\title{
Bone Grafts in Jaw Cysts- Hydroxyapatite \& Allogenic Bone - A Comparative Study
}

\author{
Showkat Mamun, Mahmuda Akhter, Motiur Rahman Molla \\ Department of Oral and Maxillofacial Surgery, Faculty of Dentistry, Bangabandhu Sheikh Mujib Medical University.
}

\begin{abstract}
:
Background: Auto bone is the gold standard in bone grafting. However, the morbidity and additional surgical time associated with its collection, as well as the limited supply, have stimulated the search for substitutes. Allograft is more limited than autograft because it yields more variable clinical results. Composite synthetic grafts offer an alternative because Hydroxyapatite is chemically identical to the inorganic matrix of living bones and it can be processed synthetically. The intent was to evaluate these two graft materials for clinical use and to provide an insight on the different grafting strategies to enhance bone formation. Objective: To find out the bone healing process and the prognostic value for the patient using hydroxyapatite alloplastic material and allogenic bone graft. Method: Total 28 patients were included in the study after the clinical and radiological evaluation where 14 cases were treated with allogenic-bone graft and rest 14 cases were treated with hydroxyapatite alloplastic material after enucleation of the cystic lesion in random manner. The integration of hydroxyapatite and allogenic bone was assessed with postoperative lesion diameter, trabecular pattern, histopathological and scintigraphic examination of the successful graft cases. Statistical analysis was carried out by 'unpaired T test' and 'Chi square' test. Result: The radiological, histopathological and scintigraphical outcome of the patients treated with hydroxyaptite granule bone graft were clinically and statistically superior in comparison with those who were treated with allogenic bone graft. Conclusion: This safe and osteoconductive hydroxyapatite appears suitable for filling bone defects and bone cavities, showing less resorption and a rapid osseous integration.
\end{abstract}

Key word: Hydroxyapatite, allogenic bone, scintigraphy, radiology, histopathology.

[BSMMU J 2009; 2(1): 25-30]

\section{Introduction:}

In maxillofacial surgery, there is always a need to fill the bony defects following surgery. So bone grafts are often necessary to provide support, fill voids, and enhance biologic repair of skeletal defects. In the orofacial region various types of bone grafts have been used that includesautografts, allografts, xenografts and alloplastic materials.

Radicular cyst is the most common type of inflammatory odontogenic cysts of the jaws, which comprises nearly $65 \%-70 \%$ of all jaw cysts ${ }^{1}$ and dentigerous cyst is the most common type of developmental odontogenic cyst of the jaw which is nearly $15 \%-18 \%$ of all jaw cysts. Following enucleation of a cyst defects smaller than $2 \mathrm{~cm}$ in diameter can be closed primarily and normally fill up with blood clot. But in case of large defect clot breakdown and infection are more likely to occur in a large haematoma. So it is considered as good technique to obliterate the dead space of a sizable defect by packing the cavity with bone or a bone substitute. In the large

Address for correspondence: Dr. Showkat Mamun, Department of Oral and Maxillofacial Surgery, Faculty of Dentistry, Bangabandhu Sheikh Mujib Medical University. Email: mamunshowkat@gmail.com surgical cavity, residual contour defects due to collapse of soft tissue into the cavity can be eliminated and the repair process appears clinically and roentgenographically to proceed more rapidly than after simple surgical excision ${ }^{2}$.

Although autogenous bone is the gold standard that all alternatives must meet or exceed, autograft has limitations, including donor site morbidity, inadequate amount, and inappropriate form ${ }^{3-5}$. These limitations have prompted increasing interest in alternative to bone grafts. Consequently, significant efforts are being made to develop ideal bone graft substitutes. ${ }^{6}$

Synthetic ceramics are readily available and are without infectious or immunogenic potential. They reduce patient morbidity significantly by reducing the need for a second operative site. Calcium phosphates (CaP) account for most ceramic-based bone graft substitutes currently available. Hydroxyapatite (HA) is the most used polycrystalline calcium phosphate ceramic mineral as an artificial bone graft substitute. It is biocompatible, highly resistant to stress forces, nontoxic and noncarcinogenic. It has marked osteoconductive and osteoinductive properties that lead to the regeneration of lamellar bone within 4 to 10 months 
of implantation. This characteristic is exploited in many surgical areas (orthopedics, maxillofacial surgery, dentistry, plastic surgery and neurosurgery). It also has been used to coat other materials to increase their stability and osteointegration.

It has been used to reconstruct defects of the jaw and other facial bones, to obliterate mastoidectomy cavities and to reconstruct the ossicular chain and wall of the outer ear canal. Other uses of Hydroxyapatite range from augmenting atrophic alveolar ridges to repairing long-bone defects, ununited fractures, middle ear prostheses, spinal fusions, and cranioplasties.

Unfortunately, the major drawbacks to the use of $\mathrm{CaP}$ ceramics are their adverse mechanical properties. They are brittle, have low impact resistance, and have low tensile strength; consequently, they fail when used in a structural capacity. The lack of viscous flow is a serious limitation for CPCs. Despite this problem, some researchers tried injecting CPCs in paste form. The most notable among such reports is that of Constantz et al ${ }^{7}$. Its tendency for granular migration and incomplete resorption has become a long-term problem ${ }^{8}$.

Allogenic bone is another attractive source of bone grafting material next to auto bone. Allogeneic bone, with variable biologic properties, is available in many preparations: frozen, freeze-dried, irradiated, or demineralized. Transplantation of allogenic bone as a method of treatment as various disorders of skeleton was started late decade of 19th century and 1st decade of 20th century. Freeze-dried bone allograft was first introduced by United State Navy tissue Bank in $1951^{9}$.

Though allografts are attractive sources, there are several problems encountered in using them, including resorbtion, relative risk of disease transmission, immunogenicity, loss of biologic and mechanical properties secondary to its processing, and non-availability world-wide due to financial and religious concerns.

Bone Trabeculae are composed of thin radiopaque plates and rods. To evaluate the trabecular pattern in a specific area it is necessary to compare with the surrounding bony trabeculae and some time with previous radiograph.

Scintigraphic images are obtained utilizing the intravenous administration of a radiopharmaceutical, particularly technetium-99m labeled diphosphonates. In an in vitro binding assay, the competitive adsorption of technetium99m labeled diphosphonates to pure hydroxyapatite is forty times greater than to pure organic bone matrix. Thus, its uptake correlates well with the rate of mineralization
${ }^{10}$. Harbert ${ }^{11}$, mentioned that bone scintigraphy provides a means of predicting graft failure before radiographic or clinical changes become apparent and, thus, helping to avoid a loss of surrounding bone from graft necrosis or infection.

Thus the study is designed to evaluate the integration of HA and allogenic bone as bone graft by assessment of postoperative lesion diameter, trabecular pattern, histopathological evaluation and scintigraphic assessment of the graft to its future use with maxillofacial reconstruction.

\section{Method:}

It was a prospective study conducted in the Department of Oral and Maxillofacial Surgery, Bangabandhu Sheikh Mujib Medical University between the period of July 2006 to June 2008. 28 patients were included in the study with inclusion criteria of non infected cystic type of lesion (Radicular and Dentigerous cyst), lesion size between 2 $\mathrm{cm}$ to $7 \mathrm{~cm}$ in diameter. Patients with any systemic bone disease or diabetes, tuberculosis, rheumatic heart diseases and renal failure, psychologically abnormal patients or patients who had taken radiotherapy in the orofacial region were excluded.

Total 28 patients were included in the study after the clinical and radiological evaluation. Among them 14 cases were treated with allogenic-bone graft and rest of the 14 cases were treated hydroxyapatite alloplastic material after enucleation of the cystic lesion in random manner.

After all preoperative investigations and radiological examination in selected cases were operated following standard surgical procedure meticulously in sterile environment under local anesthesia and sedation. Enucleation of cyst was done with removal of all granulation tissue. After enucleation of cyst bony cavity was irrigated with diluted povidone iodine for the purpose of debridment. Granules of hydroxyapatite or allobone was placed on the surgical defect and wetted with patient blood or saline solution followed by primary closure of the wound. (Fig-1, Fig-2)

The wound was checked on the following day and radiograph was done three days after operation. Sutures were removed on seventh post operative day.

\section{Assessment:}

Observation of the diameter of the lesion - The final initial radiological diameter was recorded at the 3rd post operative day to get the exact extension of the lesion because the surgical procedures have changed the preoperative lesion diameter. 
Then the Initial diameter of the lesion was measured and follow-up was done for each patient with occlusal view/ OPG or intraoral periapical view with the same magnification, exposure time, $\mathrm{Kv}$, in the same $\mathrm{X}$ ray machine with the same operator so that measurement of lesion diameter have the accurate result measured with a mm scale.

Each OPG was done by Cranex Base X (Finland) panoramic dental X-ray machine with $\mathrm{mA}$ set to 10 and $\mathrm{KV}$ set to 75 for each patient. Each occlusal view was done with mA set to 100 , KV set to 50 and exposure time set to 0.10 sec from exactly the same distance and angulation for each of the patient. For each periapical view JYF -10 (China) X-ray machine is used with $\mathrm{mA}$ was set to $10, \mathrm{KV}$ set to 55 and exposure time was set to $0.80 \mathrm{sec}$ again with maintaining the same distance and angulation. The margin of the successful bone grafts showed less sharpness than the immediate post operative margin in each case after one month.

Observation of trabecular pattern of woven boneTrabecular pattern of woven bone with each patient was evaluated visually by comparing the X-ray of the same patient from two different focuses using a standard scale with image processing software in collaboration with the Department of Radiology and Imaging, Bangabandhu Sheikh Mujib Medical University. . The first focus is from the healthy portion of bone with normal trabecular pattern and the second focus is from the intervented cystic defect with the graft which has lost the normal trabecular pattern or from the region of interest of the same patient assuming $100 \%$ trabecular pattern is present in the first focus and comparing it with the region of interest.

Histopathological examination was done after six month of operation. Few patients were randomly selected from each group both Hydroxyapatite and allobone. Tissue specimen was taken from all these cases after reflecting the mucoperiosteum at the previous lesion area to find out the presence or absence of cementing line, osteoblast, woven bone, fibrous and chronic inflammatory cell to assess the quality of the graft material in the healed lesion. This was done in the department of Pathology, BSMMU with their detailed pathological report.

Scintigram was done after six month of operation. Few patients were randomly selected from both Hydroxyapatite and allobone graft group. Three phase scan was done with technetium- 99m labeled diphosphonates for each patient to access the viability of bone graft or areas of increased bone metabolism are evidence of good viability and appear as areas of increased radiotracer uptake, namely "hot spots". Diminished or absent uptake are called "cold spots”.

\section{Data Analysis:}

In the study different variables were analyzed in all subjects including: age, sex, site of lesion, size of lesion, cortical bone status, oral hygiene radiological findings (Diameter of radiolucency and trabecular pattern after 1 , 3 and 6 months), histologic examination of the cystic cavity margin after 6 months of operation, scintigraphic findings (Evaluation of tracer uptake after 6 months of operation)

All the data sheet of history and x-ray were collected and analyzed scientifically and computer based statistical analysis using SPSS software was carried out with appropriate techniques and systems. Results were presented as a detailed pathology report that includes highresolution digital photomicrographs with image processing software. Data were analyzed statistically by 'unpaired $t$ test' and ' chi square' test.

\section{Results:}

The mean diameter of lesion was $3.3 \pm 0.98 \mathrm{~cm}$ in group A (with Hydroxyapatite bone graft) and $3.9 \pm 1.40 \mathrm{~cm}$ in group $\mathrm{B}$ (with Allogenic bone graft) during preoperative period. After 1 month the mean diameter declined and found $2.67 \pm 0.89 \mathrm{~cm}$ in group A and $2.80 \pm 0.99 \mathrm{~cm}$ in group B. After 3 months the mean diameter further reduced and found $1.92 \pm 0.63 \mathrm{~cm}$ in group A and $2.00 \pm 0.77 \mathrm{~cm}$ in group B. Finally after 6 months the mean diameter was $0.96 \pm 0.23$ $\mathrm{cm}$ in group A and $1.25 \pm 0.45 \mathrm{~cm}$ in group $\mathrm{B}$. The difference was statistically significant $(\mathrm{p}<0.05)$ after 6 months but after 1 month and 3 months the difference was not statistically significant ( $\mathrm{p}>0.05)$ (Table-I).

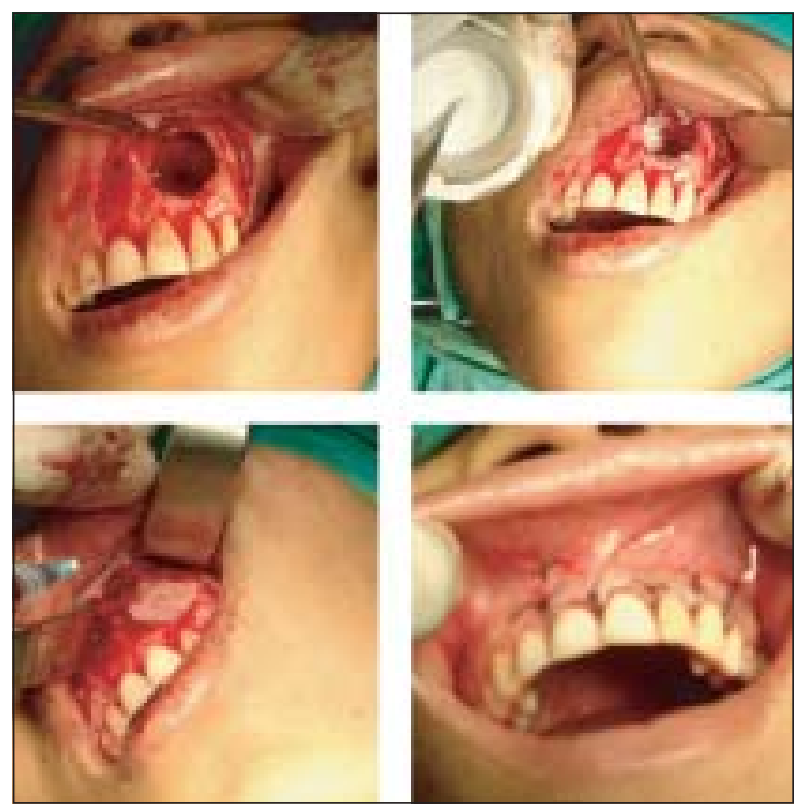

Fig.-1: Surgical procedure of hydroxyapatite bone graft in a cystic cavity. 

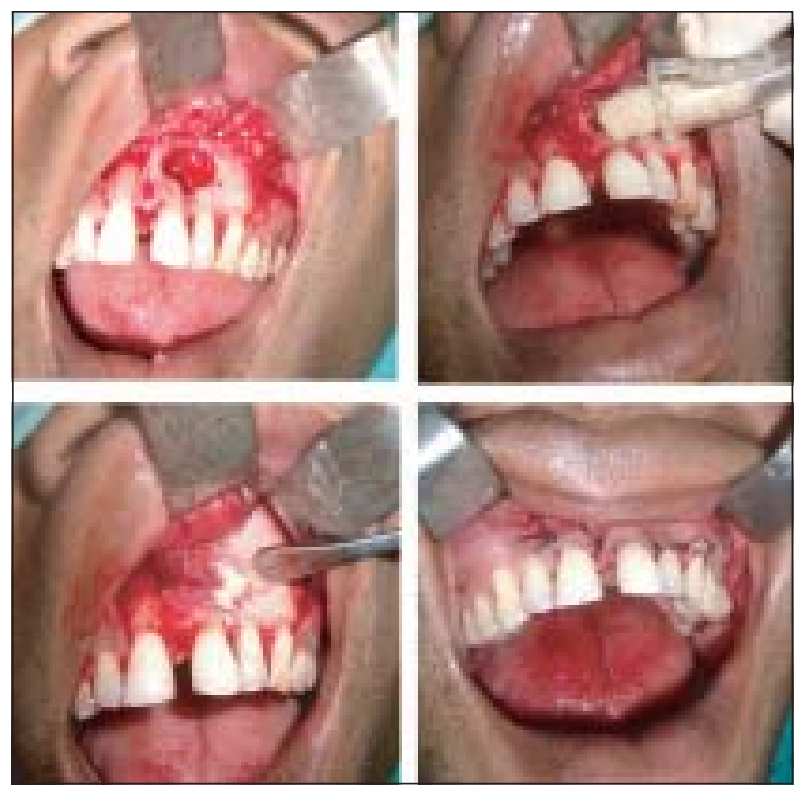

Fig.-2: Surgical procedure of allogenic bone graft in a cystic cavity.

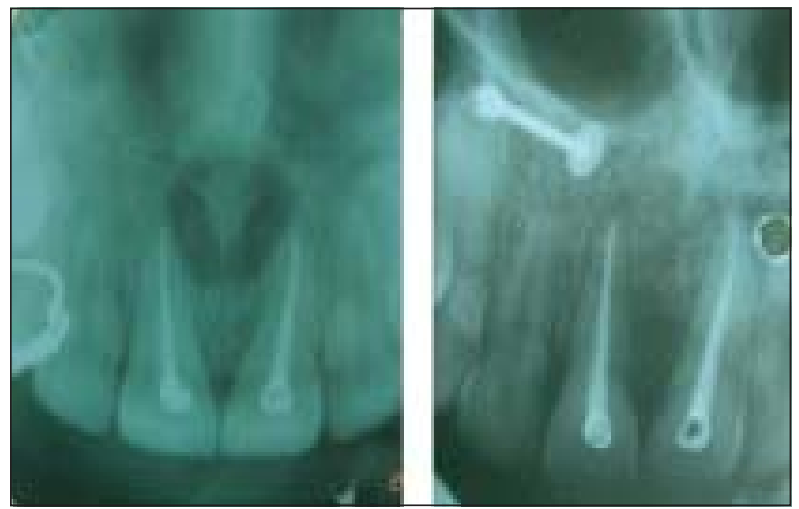

Fig. -3: Radicular cyst, treated with Hydroxyapatite bone graft. Significant radiological improvement was observed.

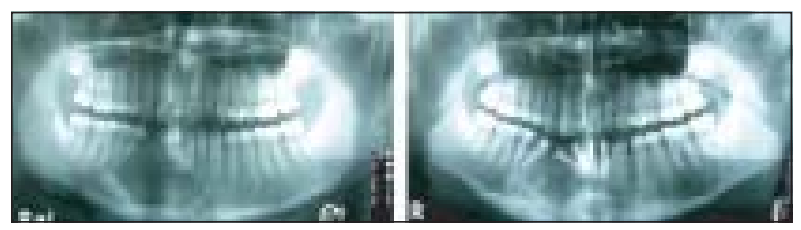

Preoperative view

Post operative view after 6 months

Fig.-4: Dentigerous cyst treated with freeze dried allogenic bone graft. Significant radiological improvement was not observed.

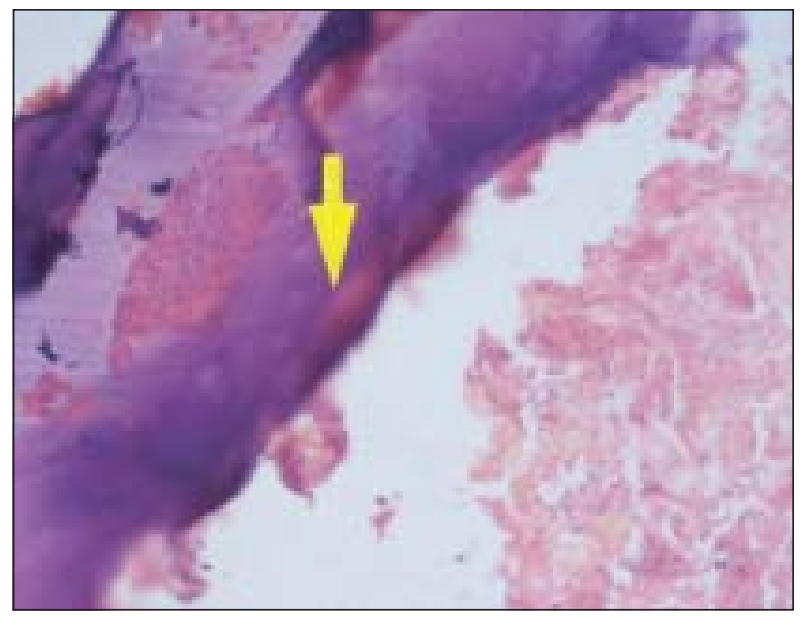

Fig.- 5: Patient treated with hydroxyapatite bone graft. Under microscope with high magnification shows osteoblastic activity and cementing line which indicates woven bone formation.



Fig. -6: Patient treated with allogenic bone graft. Under microscope with high magnification shows fibrous tissue deposition and chronic inflammatory cell which indicates no new bone formation.

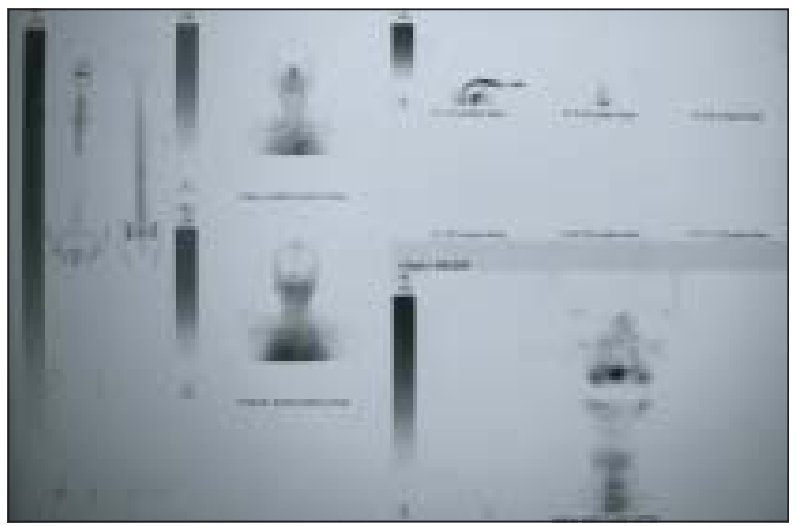

Fig.-7: Patient treated with freeze dried allogenic bone graft. Radionuclide bone scanning shows less tracer uptake which indicates average osteoblastic activity. 


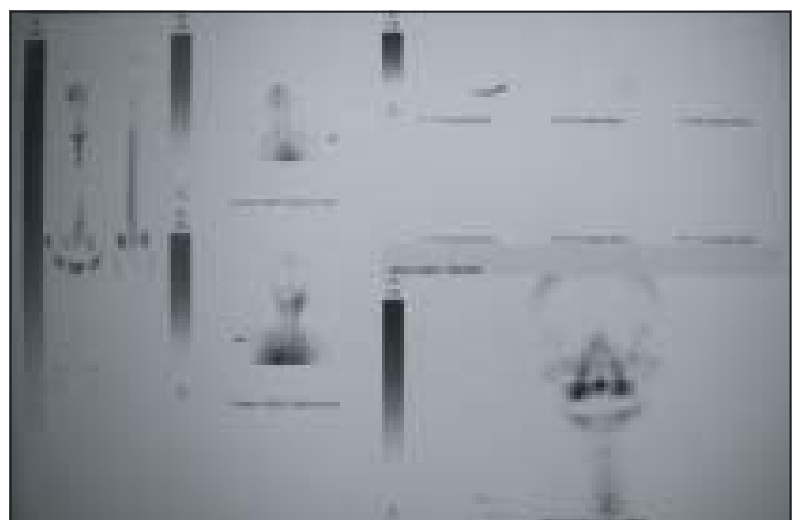

Fig.-8: Patient treated with hydroxyapatite bone graft. Radionuclide bone scanning shows increased tracer uptake which indicates good osteoblastic activity.

Table-I

Mean lesion diameter of the patients between two groups

\begin{tabular}{lccc}
\hline & $\begin{array}{c}\text { Group-A } \\
(\mathrm{n}=14)\end{array}$ & $\begin{array}{c}\text { Group-B } \\
(\mathrm{n}=14)\end{array}$ & p value \\
& Mean \pm SD & Mean \pm SD & \\
\hline Preoperative & $3.30 \pm 0.98$ & $3.90 \pm 1.40$ & $0.167^{\mathrm{NS}}$ \\
After 1 month & $2.67 \pm 0.89$ & $2.80 \pm 0.99$ & $0.725^{\mathrm{NS}}$ \\
After 3 months & $1.92 \pm 0.63$ & $2.00 \pm 0.77$ & $0.770^{\mathrm{NS}}$ \\
After 6 months & $0.96 \pm 0.23$ & $1.25 \pm 0.45$ & $0.039^{\mathrm{S}}$ \\
\hline
\end{tabular}

Group-A: With Hydroxyapatite bone graft

Group-B: With Allogenic bone graft

NS= Not significant, $\mathrm{S}=$ Significant,

$\mathrm{P}$ value reached from unpaired t-test

\section{Table-II}

Distribution of the patients by management type and incidence of infection.

\begin{tabular}{lccccc}
\hline Post operative & $\begin{array}{c}\text { Group-A } \\
(\mathrm{n}=14)\end{array}$ & $\begin{array}{c}\text { Group-B } \\
(\mathrm{n}=14)\end{array}$ & p value \\
Infection & number & $\%$ & number $\%$ & \\
\hline Infection occur & 0 & 0.0 & 5 & 35.7 & \\
No infection & 14 & 100.0 & 9 & 64.3 & $0.020^{\mathrm{S}}$ \\
Total & 14 & 100 & 14 & 100 & \\
\hline
\end{tabular}

Group-A: With Hydroxyapatite bone graft

Group-B: With Allogenic bone graft

$\mathrm{S}=$ Significant

$\mathrm{P}$ value reached from Chi square test

\section{Discussion:}

It was found that in reducing the diameter of lesion with hydroxyapatite bone grafts were both clinically and radiologically better than allogenic bone graft (Fig-3). It was supported by Zuev et $\mathrm{al}^{12}$. This observation was concluded by them that the patients with hydroxapatite bone graft showed better radiological outcome than their allogenic counterparts. They also revealed the outcomes of two groups, poorly defined in the initial few weeks but in the later period like three months it was significant, this result is very close to Schwartz et al. ${ }^{13}$

Patients with hydroxapatite bone graft showed superior trabecular pattern than the allogenic counterparts (Fig-4). The difference of trabecular pattern between the two groups started to amplify in the post operative period. The difference was almost persistent throughout the period of 6 months, with gradual increasing tendency.

Histopathological slide was prepared from randomly selected cases of both study and control group from same site and procedure after six months of operation. According to the histopathological findings the neo-osteogenetic process was better in the hydroxyapatite group (Fig. 5, Fig. 6). Microscopic findings revealed that the graft was seated in the duel process of osteoclastic and osteoblastic activities with the ingrowths of capillaries. There was marked cementing line which indicates deposition of woven bone; on the other hand there was less definite histological feature of active bone formation rather fibrous deposition showed in allogenic bone group. Moreover the infiltration of chronic inflammatory cell was evident in allo group.

Radionuclide bone scanning was done from randomly selected cases of both groups. More increased tracer uptake was seen in the operation site of hydroxyapatite group than that of in the allo group (Fig. 7, Fig. 8) which is also supported by the finding of Jun ${ }^{14}$. More tracer uptake of radioactive iodine indicates good osteoblastic activity. On the other hand slight tracer uptake indicates average or poor osteoblastic activity.

Post operative infection was not found in hydroxyapatite bone graft group and 5(35.7\%) in allogenic bone graft group and the difference was statistically significant $(\mathrm{p}<0.05)$. Infection occurred in $1(20.0 \%)$ in maxilla and $4(80.0 \%)$ in mandible (Table-II).

The infection was remarkably less in the hydroxyapatite group because of its fine granular structure which contributed to very reduced anatomical dead space than that of the allografts which had larger particles which 
contributed to infection. After placement of hydroxyapatite as a graft it solidifies and work as single unit of osseointegrated structure that also contributes to less infection than the allo group which is supported by the findings of Zasacki ${ }^{10}$. Infection occurred usually with in the one week after operation and presented by the features of localized swelling and exudation which leads to exposure of grafted materials. This condition was then treated by removal of the graft material and curettage of necrosed tissue and use of proper antibiotics.

The allograft group was more infected than the hydroxyapatite graft group may be due to loss of biological properties secondary to its processing which was supported by Friedlander ${ }^{6}$. Other reasons for this inconsistency include variable donor age with material from older donors being less osteoinductive, how carefully the material is processed, the level of residual calcium and the final particle size of the prepared graft reported by Schwartz and coworkers ${ }^{14}$.

\section{Conclusion:}

The radiological, histopathological and scintigraphical outcome of the patients treated with hydroxyaptite granule bone graft were clinically and statistically better in comparison with those treated with allogenic bone graft. None of the patient of the hydroxyapatite group was infected. This safe and osteoconductive HA appears suitable for filling bone defects and bone cavities, showing less resorption and a rapid osseous integration.

\section{References:}

1. Stockdale CR, Chandler NP. The nature of the periapical lesion: a review of 1108 cases. J Dent 1988;16: 123-129

2. Marble H.B. Jr, Captain DC USN. Homografts of freeze-dried bone in cystic defects of the jaws. Federal dental services 1968; 26(1): 118.
3. Banwart JC, Asher MA, Hassanein RS. Iliac crest bone graft harvest donor site morbidity: A statistical evaluation. Spine 1995; 20: $1055-60$.

4. Cowley SP, Anderson LD. Hernias through donor sites for iliacbone grafts. J Bone Joint Surg Am 1983; 65: 1023-25.

5. Summers BN, Eisenstein SM. Donor site pain from the ilium: a complication of lumbar spine fusion. J Bone Joint Surg Br 1989; 71: $677-80$.

6. Freidlaender GE. Immune responses to osteochondral allografts Current knowledge and future directions. Clin Orthop 1983; 174: 58-68.

7. Constanz BR, Ison IC, Fulmer MT. Skeletal rapair by in situ formation of the mineral phase of bone Science 1995; 267: 179699.

8. Rosen HM, McFarland MM. The biologic behaviour of hydroxyapatite implanted into the maxillofacial skeleton. Plast Reconstr Surg 1990; 85: 718-23

9. Zasacki W. The efficacy of application of lyophilized, radiation sterilized bone graft in orthropaedic surgery. Clin Orthro Relt Res 1991; 272: 82-87.

10. Francis MD, Horn PA, Tofe AJ. Controversial mechanism of technetium-99m deposition on bone. J Nucl Med 1981; 22: 72.

11. Harbert JC. The musculoeskeletal system In: Harbert JC, Eckelman WC, Newmann RD (eds). Nuclear medicine: diagnosis and therapy. New York: Thieme 1996; 801-63

12. Zuev VP, Dmitrieva LA, Pankratov AS. The comparative characteristics of stimulators of reparative osteogenesis in the treatment of periodontal diseases [Article in Russian]', Stomatologiia (Mosk) 1996; 75(5): 31-34.

13. Schwartz z. Ability of commercial demineralized freeze- dried bone allograft to induce new bone formation. J Periodontol 1996; 67( 9): 925- 30.

14. Jun Manabe Pasteurized autologous bone graft in surgery for bone and soft tissue sarcoma J Clin Orthop 2004; 419: 258. 